\title{
Comparison of the characteristic energy of precipitating electrons derived from ground-based and DMSP satellite data
}

\author{
M. Ashrafi, M. J. Kosch, and F. Honary \\ Department of Communications Systems, Lancaster University, Lancaster, LA1 4WA, UK \\ Received: 15 December 2003 - Revised: 16 April 2004 - Accepted: 5 May 2004 - Published: 31 January 2005 \\ Part of Special Issue "Eleventh International EISCAT Workshop"
}

\begin{abstract}
Energy maps are important for ionospheremagnetosphere coupling studies, because quantitative determination of field-aligned currents requires knowledge of the conductances and their spatial gradients. By combining imaging riometer absorption and all-sky auroral optical data it is possible to produce high temporal and spatial resolution maps of the Maxwellian characteristic energy of precipitating electrons within a $240 \times 240 \mathrm{~km}^{2}$ common field of view. These data have been calibrated by inverting EISCAT electron density profiles into equivalent energy spectra. In this paper energy maps produced by ground-based instruments (optical and riometer) are compared with DMSP satellite data during geomagnetic conjunctions. For the period 1995-2002, twelve satellite passes over the groundbased instruments' field of view for the cloud-free conditions have been considered. Four of the satellite conjunctions occurred during moderate geomagnetic, steady-state conditions and without any ion precipitation. In these cases with Maxwellian satellite spectra, there is $\sim 71 \%$ agreement between the characteristic energies derived from the satellite and the ground-based energy map method.
\end{abstract}

Key words. Ionosphere (particle precipitation; ionospheremagnetosphere interaction; auroral ionosphere)

\section{Introduction}

Precipitating auroral particles with different energies are deposited at different altitudes where they excite atoms and molecules which cause auroral optical emissions. Precipitating particles are also responsible for ionospheric radio absorption.

Correlation between auroral luminosity and ionospheric radio absorption has been well known for many years

Correspondence to: M. Ashrafi

(m.ashrafi@lancaster.ac.uk)
(Heppner et al., 1952; Campbell and Leinbach, 1961). Holt and Omholt (1962) and Gustafsson (1969) found a good correlation between the absorption and the intensity fluctuation of the auroral green line $(557.7 \mathrm{~nm})$. Ansari (1964) argued that there is a peak-to-peak correspondence between absorption fluctuations and intensity fluctuation of $557.7 \mathrm{~nm}$ in the pre-midnight hours while a significant increase in the ratio of absorption to optical intensity in the post-midnight hours is an indication of a hardening of the primary precipitating electron energy spectrum. Other studies include inferring the energy spectrum of the auroral electrons using simultaneous measurements with photometers and riometers (Johansen, 1965; Berkey, 1968). These observations, in conjunction with measurements from satellite and rocket experiments, have shown auroral emissions and absorption relate to the flux and energy characteristics of auroral precipitating electrons. This relationship has been examined both experimentally (Vondrak and Sears, 1978; Collis et al., 1984; Steele and McEwen, 1990; Robinson and Vondrak, 1994) and by computational modelling (Rees and Luckey, 1974). However, all these studies were done with narrow fields of view.

Using imaging riometer and optical imager data, highresolution maps of the characteristic energy of auroral particles were produced (Kosch et al., 2001a). This approach allows for the study of small-scale spatial and temporal morphology of the auroral particle energies over a wide field of view. The technique is important because it offers a new and quantitative way of viewing particle precipitation which includes energy information.

Assuming the same source population for hard and soft electron precipitation, Kosch et al. (2001a) showed that the characteristic Maxwellian energy of precipitating electrons is a function of D-layer auroral absorption (riometer data) divided by the square root of E-layer auroral optical intensity (557.7 nm data). Electron density height profiles from incoherent scatter radar data can be used with an atmospheric model to estimate the spectrum of precipitating 
electrons (del Pozo et al., 1997). EISCAT has been used to calibrate the characteristic energy of the precipitating electrons derived from optical and absorption data for an assumed Maxwellian energy spectrum.

In this paper DMSP satellite data have been used to evaluate the energy map method. On-board sensors of these satellites provide a complete energy spectrum of the low energy auroral particles. The data set consists of electron fluxes between $30 \mathrm{eV}$ and $30 \mathrm{keV}$ recorded every second. A Maxwellian distribution has been found to represent many observed primary auroral spectra (Rees, 1989). By fitting this distribution to satellite data the characteristic energy of auroral electrons along the satellite track can be estimated every second. Similar statistical models have been generated from early DMSP satellites to obtain global maps of the energy flux of auroral electron precipitation (Hardy et al., 1985). By mapping down the satellite geomagnetic coordinates into the common field of view of the imaging riometer and all-sky imager we compare the characteristic energy estimate from the satellite data with that derived from the ground-based energy maps.

To understand magnetospheric-ionospheric coupling, temporal and spatial mapping of the ionospheric conductances is required. Ionospheric conductances and electric fields are needed to derive the field-aligned current systems responsible for energy transfer between the magnetosphere and the ionosphere. Therefore, considerable effort has been devoted to the estimation of the ionospheric conductances from measurable geophysical parameters.

Kosch et al. (1998b) showed that, by using a TV auroral imager at $557.7 \mathrm{~nm}$, one can obtain large spatial scale images with high temporal and spatial resolution for estimating the distribution of nocturnal ionospheric Pedersen conductance. The method works because Pedersen conductance and the 557.7-nm optical emission both maximise in the E-region. The measurement was calibrated using EISCAT data at one point and then applied to the camera's entire field of view. However, measuring the Hall conductance is more difficult since the Hall current occurs at lower altitudes and is therefore much more dependent on the energy of the precipitating particles (Kosch et al., 1998b). Having a precise map of the precipitating electrons' characteristic energy, it is possible to calculate Hall conductance from the Pedersen conductance, knowing that the ratio of Hall to Pedersen conductance is an indication of the average energy (Vickery et al., 1981). In addition, it is well understood that computing field-aligned currents relies on electric field distributions, as well as Pedersen and Hall conductances' distribution and their spatial gradients. Kosch et al. (2001b) has shown that assuming spatially uniform conductances and ignoring the gradients leads to gross errors. Both ionospheric Hall and Pedersen conductances, as well as their gradients, can be calculated with high temporal and spatial resolution using the groundbased method as explained in greater detail in the following sections.

\section{Instrumentation}

\section{$2.1 \quad$ DMSP}

The DMSP (Defence Meteorological Satellite Program) sunsynchronous satellites, with 101 min of near-polar orbits at a nominal altitude of $830 \mathrm{~km}$, provide global monitoring of the solar terrestrial environment. These satellites cover the entire polar region at least twice, and the equatorial region once a day. The SSJ/4 sensor, which has been used in this study, is a precipitating Electron and Ion Spectrometer, consists of four curved plate electrostatic analysers which measure the flux of charged particles with energies between $30 \mathrm{eV}$ and $30 \mathrm{keV}$ as they flow past the spacecraft toward the Earth. The resulting counts are converted into differential number flux. The satellites are three axis stabilised with particle detectors pointing toward local zenith, allowing precipitating electrons and ions to enter through an aperture of $8^{\circ}$ field of view (Hardy et al., 1984). Each pair of high and low energy detectors consist of 10 logarithmically-based energy channels which dwell on each channel for $0.09 \mathrm{~s}$ from high energy to low energies, such that once a second a complete spectrum over the entire energy range is produced (Hardy et al., 1985). The loss cone for polar orbiting satellites at DMSP altitudes is $\sim 50^{\circ}$ and the SSJ/4 provide measurements within pitch angles $<16^{\circ}$, which means that, at the latitudes of the interest to the present research, only particles well within the loss cone are observed. The measured spectra are the average of the real spectra within the loss cone at the DMSP altitude.

\subsection{IRIS}

Data from Imaging Riometer for Ionospheric Studies (IRIS) at $69.05^{\circ} \mathrm{N}, 28.79^{\circ} \mathrm{E}$, northern Finland, have been used for producing energy maps. IRIS records ionospheric absorption of cosmic noise at $38.2 \mathrm{MHz}$ measured by its 49 beams every second. Primary auroral particles with energies between a few tens $\mathrm{keV}$ and a few hundred $\mathrm{keV}$ cause ionospheric radio wave absorption at the D-region, with a peak of absorption around $90 \mathrm{~km}$ (Hargreaves, 1995). The projection of IRIS beams at this height spans an area of $67.8^{\circ}-70.2^{\circ} \mathrm{N}$ and $17.75^{\circ}-23.75^{\circ} \mathrm{E}$, which covers approximately $240 \times 240 \mathrm{~km}^{2}$. IRIS is capable of producing absorption images with 1-s temporal resolution continuously with a sensitivity of $\sim 0.01 \mathrm{~dB}$. A detailed description of imaging riometer techniques and IRIS characteristics can be found in Stauning (1996) and Browne et al. (1995), respectively.

\subsection{DASI}

The Digital All-Sky Imager (DASI) (Kosch et al., 1998a) located at $69.35^{\circ} \mathrm{N}$ and $20.36^{\circ} \mathrm{E}$, northern Norway, is an automatic low-light-level television camera system coupled to a telecentric all-sky lens producing integrated optical images every $10 \mathrm{~s}$. DASI operates only during the winter and for all dark and moon-free periods. Most of the auroral luminosity is due to the green-light emission at $557.7 \mathrm{~nm}$ from the ${ }^{1} S$ state of atomic oxygen (Vallance-Jones, 1991), with a 
maximum intensity at around $100-110 \mathrm{~km}$ altitude (Störmer, 1955). DASI uses a narrow band $(2.5 \mathrm{~nm})$ interference filter at $557.7 \mathrm{~nm}$ and is calibrated in Rayleighs. The circular allsky projection at $100 \mathrm{~km}$ altitude is transformed to an area of $67.6^{\circ}-72.6^{\circ} \mathrm{N}$ and $13.5^{\circ}-26.0^{\circ} \mathrm{E}$, which covers a square of $520 \times 520 \mathrm{~km}^{2}$ with a spatial resolution of $10 \times 10 \mathrm{~km}^{2}$.

\section{$2.4 \quad$ SCASI}

To ensure that DASI measures the real optical intensity, the Skibotn CCD All-Sky Imager, SCASI (Kosch, 1999) has been used for cloud cover detection. SCASI is a slow-scan CCD imager with an all-sky dual mirror system which takes a full spatial resolution image in white light with a 30 s exposure every $3 \mathrm{~min}$. Computer algorithms automatically assess SCASI image data and determine cloud free periods (Seviour et al., 2003).

\section{Background theory}

According to Ohm's law the height integrated ionospheric current $(\mathrm{J})$ can be written as:

$\boldsymbol{J}=\Sigma_{P} \boldsymbol{E}+\Sigma_{H}(\hat{\boldsymbol{B}} \times \boldsymbol{E})$,

where $\boldsymbol{E}$ is the electric field vector and $\hat{B}$ is the magnetic field vector in the direction of the Earth's magnetic field. $\Sigma_{P}$ and $\Sigma_{H}$ are the Pedersen and Hall conductances, respectively, height integrated between 90 and $170 \mathrm{~km}$ (Brekke et al., 1974). Assuming a vertical magnetic field we compute field-aligned currents $\left(J_{\|}\right)$as follows:

$\boldsymbol{J}_{\|}=\operatorname{div} \boldsymbol{J}=\operatorname{div}\left(\Sigma_{P} \boldsymbol{E}\right)+\operatorname{div}\left(\Sigma_{H}(\hat{\boldsymbol{z}} \times \boldsymbol{E})\right)$.

Rearranging and assuming that $\mathrm{E}$ is a curl-free electric field, gives:

$J_{\|}=\Sigma_{P} \operatorname{div} \boldsymbol{E}+\nabla \Sigma_{P} \cdot \boldsymbol{E}+\nabla \Sigma_{H} \cdot(\hat{\boldsymbol{z}} \times \boldsymbol{E})$.

The important consequence of this equation is that $J_{\|}$is determined by ionospheric conductances and electric fields. One straight-forward description is that auroral precipitating particles are responsible for producing auroral emissions as well as enhanced ionisation in the upper atmosphere and therefore ionospheric conductivity. In addition, particle precipitation results in conductivity gradients, which can be highly variable in time and occur in small-scale structures (e.g. $1-20 \mathrm{~km}$ ). The electric fields in the immediate vicinity of auroral precipitation are strongly modified by these conductance variations. Measurements of electric fields, currents and conductivities covering a large area in the ionosphere with high spatial resolution are not easily possible.

Using energetic electron fluxes measured by instruments carried by polar orbiting satellites, Robinson et al. (1987) and Fuller-Rowell and Evans (1987) derived height-integrated Hall and Pedersen conductivities. Auroral zone conductances can be estimated from the energy flux and average energy of precipitating electrons (Robinson et al., 1987). Empirical equations relating the Hall and Pedersen conductances to the average energy $(\bar{E})$ and energy flux $\left(\phi_{E}\right.$ in $\left.\mathrm{ergs} / \mathrm{cm}^{2} \mathrm{~s}\right)$ are as follows:

$$
\begin{aligned}
& \Sigma_{P}=\frac{40 \bar{E}}{16+\bar{E}^{2}} \phi_{E}^{1 / 2} \\
& \frac{\Sigma_{H}}{\Sigma_{P}}=0.45(\bar{E})^{0.85} .
\end{aligned}
$$

However, satellite methods cannot provide sufficient spatial coverage on demand to study the highly dynamic auroral structures. The method of characteristic energy maps, described below in more detail, solves this problem. Equation (5) reinforces the notion, introduced before, that estimating the precipitating particle energy will yield the Hall conductance, provided the Pedersen conductance is known, which is possible according to the earlier work described by Kosch et al. (1998b).

Primary auroral electrons can have a wide range of energy distributions. The energy spectra can be complex due to the nature of various particle acceleration mechanisms in the magnetosphere. However, many remote sensing techniques have suggested that it is sufficient to approximate the spectra by:

$F(E)=E^{\gamma} \exp \left(-E / E_{0}\right)$

in which $E_{0}$ is known as characteristic energy (Rees, 1989). For $\gamma=0$, Eq. (6) gives a exponential function, whereas $\gamma=1$ describes a Maxwellian and $\gamma \geq 2$ is a modified power-law. An isotropic pitch angle Maxwellian velocity distribution produces a Maxwellian energy distribution while an unidirectional Maxwellian velocity distribution with a very narrow pitch angle dispersion produces the exponential energy spectrum (del Pozo et al., 2002). The so-called modified power law is caused by an electron population in the tail of the Maxwellian energy distribution which has been accelerated along the field lines after the particle pitch angle was already in the loss cone (Rostoker et al., 1985). Ignoring the low energy part of the spectrum (typically $<1 \mathrm{keV}$ ) for the Maxwellian leads to an exponential spectrum. The electron fluxes less than $100 \mathrm{eV}$ consist of secondary electrons whose spectra are more likely to be described by a power-law (Ogilvie, 1968; Frank and Ackerson, 1971). In this paper we have assumed a Maxwellian spectrum for the DMSP satellite electron flux data in order to be consistent with the assumption made in producing the ground-based energy maps by Kosch et al. (2001a), which has been described in detail by these authors.

In producing energy maps with the data from IRIS and DASI, the electron continuity equation in the E-region is:

$\frac{d N_{e}}{d t}=Q_{p}+Q_{s}-\alpha N_{e}^{2}-\operatorname{div}\left(N_{e} \cdot \bar{v}\right)$,

(Brekke et al., 1989), where $N_{e}$ is the electron density, $Q_{p}$ is the ion production rate due to auroral precipitation, $Q_{s}$ is the ion production rate due to solar radiation, $\bar{v}$ is the electron bulk velocity and $\alpha$ is the effective ion recombination coefficient. For nighttime $\left(Q_{s}=0\right)$ under steady-state conditions 
Table 1. DMSP satellite conjunctions.

\begin{tabular}{|c|c|c|c|c|c|c|c|}
\hline (1) & (2) & (3) & $(4)$ & (5) & (6) & (7) & (8) \\
\hline Date & $\begin{array}{l}\text { Time(UT)- } \\
\text { Duration }\end{array}$ & DMSP & $K_{p}$ & $\begin{array}{c}\text { Ion } \\
\text { precipitation }\end{array}$ & $\begin{array}{c}\text { Aurora } \\
\text { steady state }\end{array}$ & $\begin{array}{l}\text { No. of satellite } \\
\text { spectra below } \\
\text { threshold }(0.5)\end{array}$ & $\begin{array}{c}\text { Satellite-ground- } \\
\text { based disagreement }\end{array}$ \\
\hline 18 Feb. 1995 & $18: 26: 13-31 \mathrm{~s}$ & F12 & 3 & $\mathrm{Y}$ & $\mathrm{N}$ & $90 \%$ & $236 \%$ \\
\hline 16 Oct. 1996 & $18: 29: 21-29 \mathrm{~s}$ & F12 & 2 & Y & $\mathrm{Y}$ & $93 \%$ & $35 \%$ \\
\hline 7 Jan. 1997 & $18: 36: 07-29 \mathrm{~s}$ & $\mathrm{~F} 12$ & $2^{+}$ & $\mathrm{Y}$ & $\mathrm{N}$ & $52 \%$ & $304 \%$ \\
\hline 9 Feb. 1997 & $18: 40: 46-30 \mathrm{~s}$ & F12 & $5^{+}$ & $\mathrm{N}$ & $\mathrm{N}$ & $26 \%$ & $27 \%$ \\
\hline 6 Nov. 1997 & $17: 35: 32-31 \mathrm{~s}$ & F14 & 4 & $\mathrm{~N}$ & $\mathrm{~N}$ & $16 \%$ & $164 \%$ \\
\hline 22 Nov. 1997 & $17: 39: 59-30 \mathrm{~s}$ & F14 & $4^{+}$ & $\mathrm{N}$ & $\mathrm{N}$ & $47 \%$ & $186 \%$ \\
\hline 19 Feb. 1998 & $18: 25: 40-30 \mathrm{~s}$ & F12 & $2^{+}$ & Y & $\mathrm{Y}$ & $53 \%$ & $428 \%$ \\
\hline 6 Dec. 1999 & $17: 59: 27-27 \mathrm{~s}$ & F12 & 3 & $\mathrm{~N}$ & $\mathrm{Y}$ & $30 \%$ & $31 \%$ \\
\hline 6 Jan. 2000 & $18: 20: 18-31 \mathrm{~s}$ & F15 & 3 & $\mathrm{~N}$ & $\mathrm{Y}$ & $61 \%$ & $21 \%$ \\
\hline 28 Nov. 2000 & $15: 19: 46-32 \mathrm{~s}$ & F13 & $4^{+}$ & $\mathrm{Y}$ & $\mathrm{Y}$ & $50 \%$ & $104 \%$ \\
\hline 1 Dec. 2000 & $18: 42: 28-31 \mathrm{~s}$ & F15 & $1^{-}$ & $\mathrm{Y}$ & $\mathrm{N}$ & $52 \%$ & $107 \%$ \\
\hline 23 Jan. 2001 & $17: 11: 08-31 \mathrm{~s}$ & F12 & $3^{+}$ & $\mathrm{N}$ & $\mathrm{Y}$ & $97 \%$ & $49 \%$ \\
\hline
\end{tabular}

$\left(\frac{d N_{e}}{d t}=0\right)$ and negligible transport $\left(N_{e} \cdot \bar{v}=0\right), Q_{p}$ relates to the square of the electron density:

$Q_{p}=\alpha N_{e}^{2}$.

Since auroral optical luminosity $(I)$ is proportional to $Q_{p}$ :

$N_{e} \propto \sqrt{I}$

Height integrated ionospheric absorption $(A)$ depends on Dregion electron density, as well as electron collision frequency (Hargreaves, 1969). To first order D-region auroral absorption is proportional to electron density:

$A(d B) \propto N_{e}$.

Electrons with energies $E_{A}>25 \mathrm{keV}$ deposit in the D-region (below $\sim 95 \mathrm{~km}$ ), and electrons with energies $E_{I}<15 \mathrm{keV}$ deposit in the E-region (above $\sim 100 \mathrm{~km}$ ). Knowing that the ion production rate $\left(Q_{p}\right)$ is proportional to the flux of precipitating particles $(F)$ (Rees, 1963) and assuming that the same source population for both electron precipitation causes the auroral absorption and auroral luminosity, the ratio of absorption over square root of the optical intensity is an energy indicator for the precipitating electrons (Kosch et al., 2001a):

$\frac{A}{\sqrt{I}} \propto \frac{N_{e}(H<96 \mathrm{~km})}{N_{e}(H>101 \mathrm{~km})} \propto \frac{\sqrt{F(E>25 \mathrm{keV})}}{\sqrt{F(E<15 \mathrm{keV})}}$.

Substituting Eq. (6) $\left(\frac{A}{\sqrt{I}}=\frac{\sqrt{F(E)_{A}}}{\sqrt{F(E)_{I}}}=\frac{\sqrt{k_{A} E_{A} e^{-E_{A} / E_{0}}}}{\sqrt{k_{I} E_{I} e^{-E_{I} / E_{0}}}}\right)$ and applying the logarithm to the Eq. (11):

$\ln \left(\frac{A}{\sqrt{I}}\right)=\frac{1}{2} \ln \left(\frac{k_{A}}{k_{I}}\right)+\frac{1}{2} \ln \left(\frac{E_{A}}{E_{I}}\right)+\frac{E_{I}-E_{A}}{2} \frac{1}{E_{0}}$,

which is a linear equation $(Y=C+M X)$ where $X=\frac{1}{E_{0}}$. The factor $\frac{1}{2}$ on the right-hand side of Eq. (12) corrects an error in Eq. (13) of Kosch et al. (2001a). Within the IRIS and
DASI common field of view, using absorption images from the IRIS riometer and optical images from the DASI imager, term $Y$ has been calculated. $E_{0}$ can be estimated from EISCAT by inverting its electron density profile into equivalent energy spectra at one point in the sky, thereby giving $X$. Knowing $X$ and $Y$ from Eq. (12) and using a least-squares fitting to a scatter plot of $\ln \left(\frac{A}{\sqrt{I}}\right)$ vs. $\frac{1}{E_{0}}$, the unknown terms $C$ and $M$ have been determined. By applying these two parameters to Eq. (12) in the common field of view of DASI and IRIS, high-resolution ground-based maps of characteristic energy have been obtained.

\section{Data description and analysis}

The data discussed in this study were selected from all the possible DMSP geomagnetic conjunctions within the IRIS and DASI common field of view for all cloud-free periods during 1995-2002 (Table 1). Geomagnetic conjunction occurs when the satellite ephemeris in corrected geomagnetic coordinates for the magnetic field line passing through the satellite is within the ground-based instruments' field of view in corrected geomagnetic coordinates. The IGRF-2000 model has been employed for calculating geomagnetic coordinates. DASI, with its $520 \times 520 \mathrm{~km}^{2}$ field of view includes the IRIS field of view of $240 \times 240 \mathrm{~km}^{2}$ (Fig. 1); therefore, the energy maps have been produced within the IRIS and DASI common field of view with $10 \times 10 \mathrm{~km}^{2}$ and 10 -s resolution. The satellite spatial and temporal resolution is about $0.06^{\circ}$ of orbit, corresponding to $7.5 \mathrm{~km}$ in the ionosphere, and $1 \mathrm{~s}$, respectively. The data presented here is either from the F12 or F15 DMSP satellites. F12 is oriented roughly in 09:30-21:30 LT, and F15 in the 10:30-22:30 LT meridian. Data from 12 different conjunctions investigated in this study are shown in Table 1. Columns 1-4 give the date, time, 


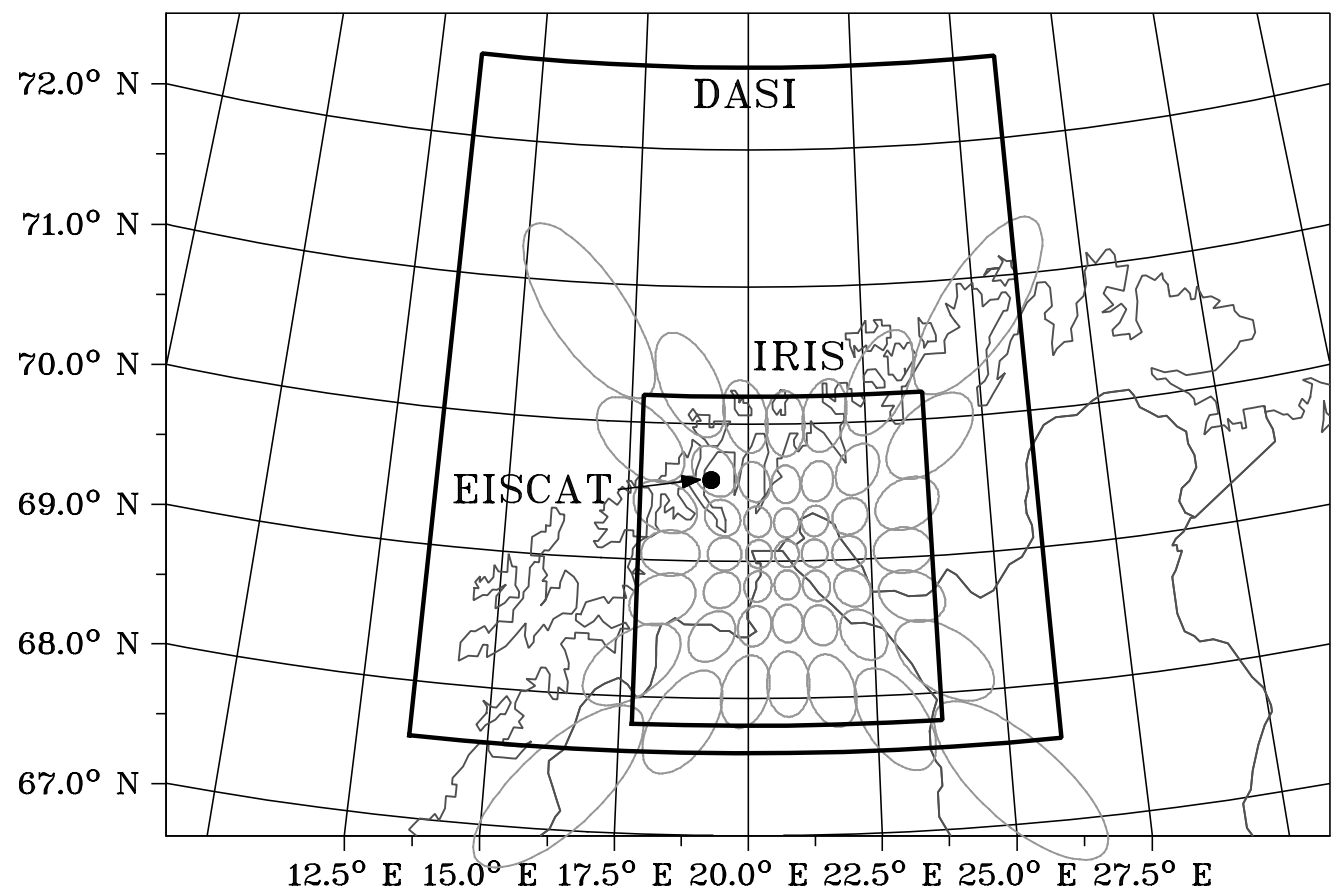

Fig. 1. A geographic map of northern Scandinavia showing the field of view of the Digital All-Sky Imager (DASI) processed data (large trapezium), the field of view of the Imaging Riometer for Ionospheric Studies (IRIS) processed data (small trapezium), the IRIS beam pattern projected up to $90 \mathrm{~km}$ altitude (ellipses), and the EISCAT radar's position projected up the magnetic field line to $100 \mathrm{~km}$ altitude.

duration of the pass, DMSP satellite number and $K_{p}$ value during the conjunction, respectively. Column 5 gives an indication whether significant proton flux was present in the DMSP data (i.e. proton flux with 1-2 orders of the magnitude of the electron flux). Column 6 gives a subjective estimate as to whether the aurora within the DASI field of view were steady state or not. This relates to the validity of the steady-state assumption of Eq. (7).

In order to evaluate the goodness of fit to the satellite spectra, we have calculated the mean fractional difference between the satellite flux data and its Maxwellian fit for each second of the satellite spectra. Assuming $F_{O i}$ is the satellite observed flux in the $i$ th energy bin and $F_{E i}$ is the expected flux derived from the Maxwellian fit to the satellite data in each energy channel, the fractional difference between the satellite flux and the fitted flux, integrated over the whole spectra is given by:

$\sum_{i} \frac{\left|F_{O i}-F_{E i}\right|}{F_{E i}}$.

Equation (13) is averaged over the number of the energy bins used in order to calculate the mean fractional difference for each second of the satellite data. We define the threshold condition for accepting the fit such that the mean fractional difference between the satellite flux data and the expected value derived from the Maxwellian fit is $<0.5$. This threshold of 0.5 will reject the satellite data that deviate by more than $50 \%$ from the Maxwellian fit, on average. Column 7 of Table 1 gives the percentage of fitted satellite spectra, whose Maxwellian function meets the threshold condition in each of the satellite conjunctions. Therefore, the higher the value in column 7 , the bigger the proportion of satellite data that meets the threshold criteria. This process is necessary for comparison to the ground-based data, which was only calibrated for Maxwellian spectra.

The purpose of this paper is to compare the derived characteristic energy from the satellite data with that of the groundbased instruments (IRIS and DASI). Comparisons have been considered only for those satellite flux spectra which have a good Maxwellian fit ( $<0.5$ mean fractional difference). Column 8 gives the average discrepancy between the characteristic Maxwellian energy computed from the ground-based energy map method and satellite data for the specific satellite flux spectra which have met the threshold condition described above, and is given by:

$\frac{\left|E_{0 S}-E_{0 G b}\right|}{E_{0 S}} \times 100$,

where $E_{0 S}$ and $E_{0 G b}$ are the Maxwellian characteristic energy derived from the satellite flux data and ground-based data, respectively. We now present three case studies, two of which were during steady-state conditions and therefore satisfy the assumptions made by the energy map technique. In the third example, this condition is not satisfied and we discuss the discrepancies between the ground-based and satellite measurements.

Figures 2 and 3 present data from 6 January 2000 and 6 December 1999, respectively. Panels (A, B and C) in each figure are absorption, optical data and the corresponding ground-based energy map, for the $40 \mathrm{~s}$ covering 
$18: 20: 10$

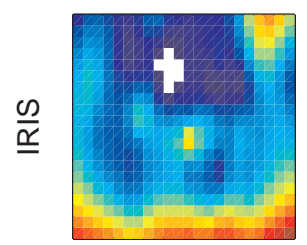

B
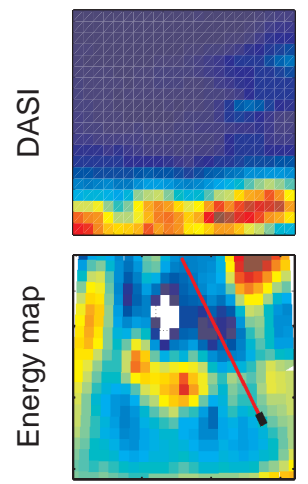

$18: 20: 20$
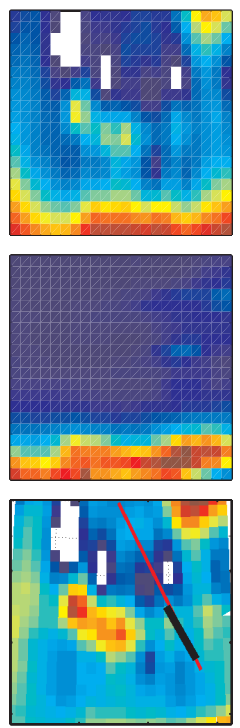

$18: 20: 30$
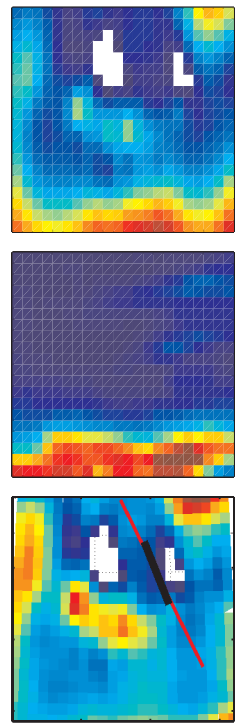

$18: 20: 40$
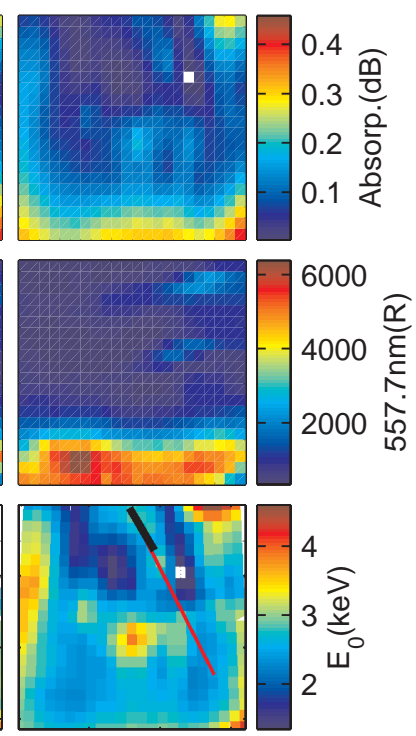

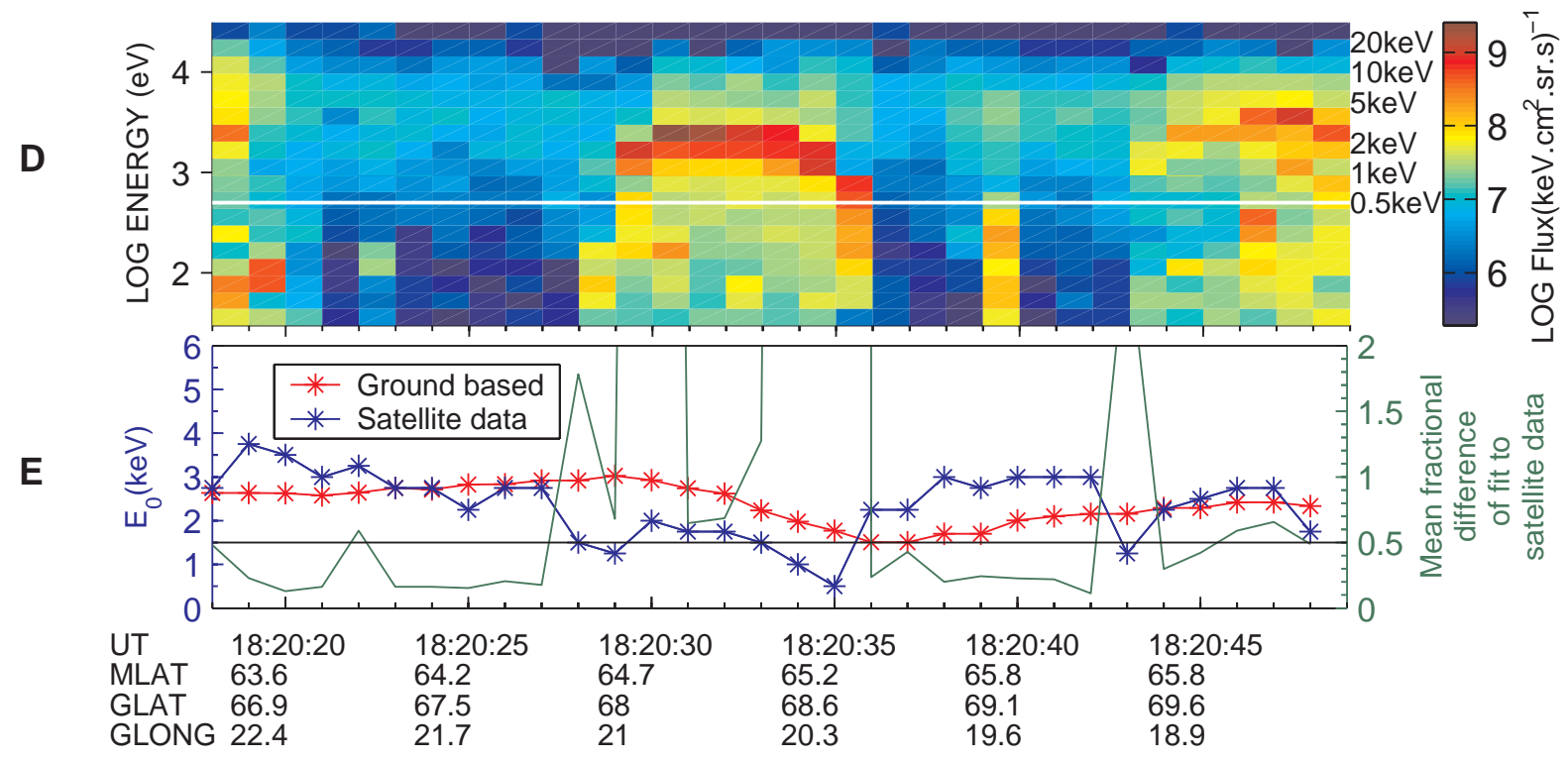

Fig. 2. Data from 6 January 2000, DMSP (F15) conjunction over northern Scandinavia $\left(K_{p}=3\right)$. Panels (A), (B) and (C) are absorption, optical intensity and energy map images, respectively, corresponding to the area covered by the IRIS and DASI common field of view $\left(67.8-70.2^{\circ} \mathrm{N}\right.$ and $\left.17.75-23.75^{\circ} \mathrm{E}\right)$ with north and east oriented up and right, respectively. Panel (D) is the satellite electron energy spectrogram. Panel (E) shows the characteristic energies derived from satellite (blue) and energy map images (red). The green curve is an indication of how non-Maxwellian the satellite spectra are.

the satellite conjunction, shown in geographic coordinates $\left(67.8^{\circ}-70.2^{\circ} \mathrm{N}\right.$ and $\left.17.75^{\circ}-23.75^{\circ} \mathrm{E}\right)$. The path of the satellite over the energy map is shown in red while the black lines indicate the part of the spacecraft path for each of the 10-s integrated images. The satellite conjunction with the groundbased instruments' field of view has been considered in geomagnetic coordinates but plotted in geographic coordinates, hence the red line start and end points are not necessarily at the image boundaries in geographic coordinates. White spaces inside the energy map images correspond to gaps in the data where an imaging riometer beam is not considered due to being affected by scintillation. Ionospheric scintillation occurs due to illumination of ionospheric irregularities by a strong point source, such as a radio star. Small-scale structures in the ionospheric electron density can cause large variations in the received signal power, which may result in unrealistic, negative absorption values in the riometer data. The effect of the scintillation sources has been eliminated by ignoring the affected beam data.

Panel (D) shows the electron energy spectrogram with flux and energy in logarithmic scales. The white line is the lower energy threshold, which has been used for estimating 


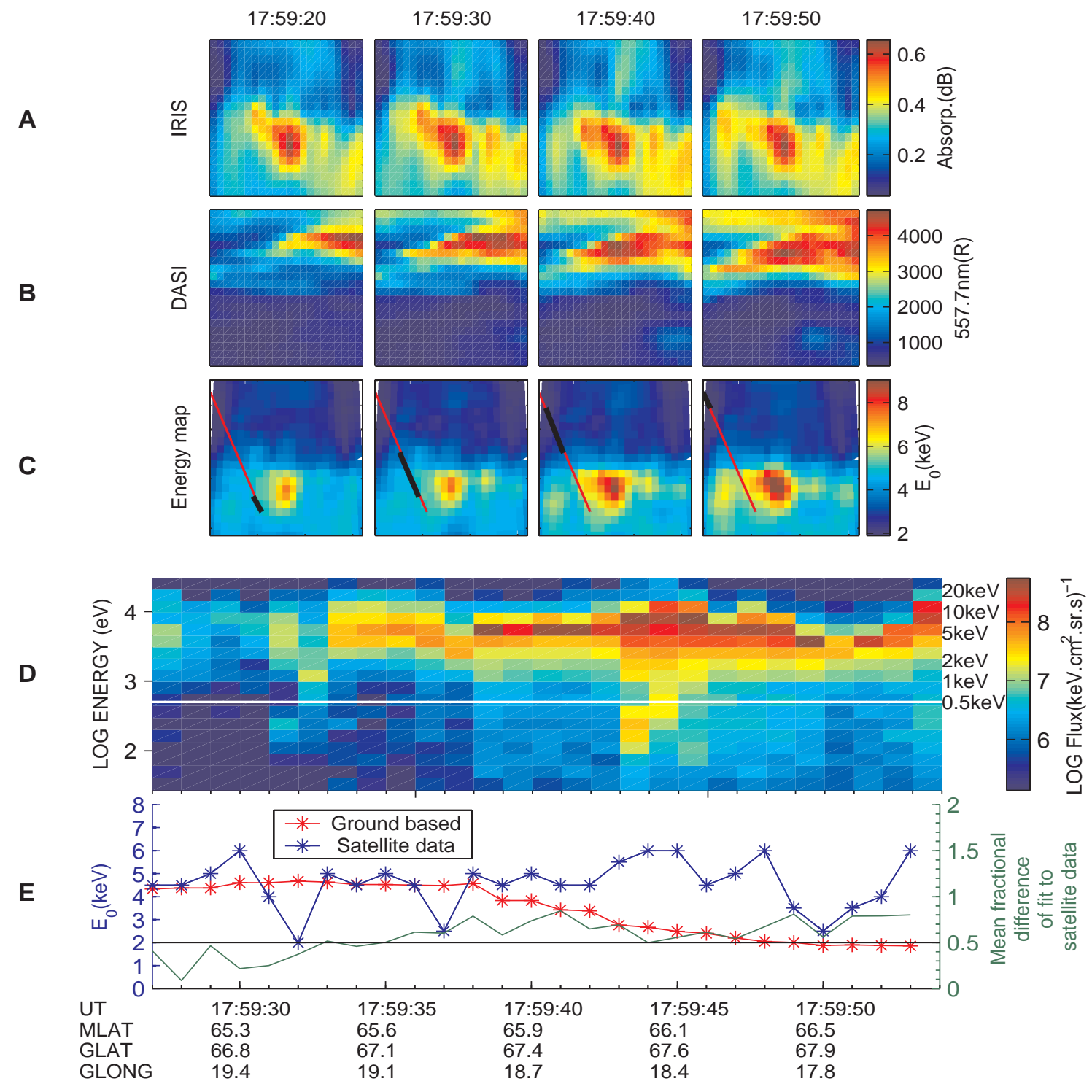

Fig. 3. Same as Fig. 2 but for 6 December 1999, DMSP (F12) and $K_{p}=3$.

the characteristic energy. This is because the energies less than $500 \mathrm{eV}$ have an insignificant contribution to the 557.7-nm optical signal and no effect on the riometer absorption data, and consequently, they do not contribute to the energy map results. Hence, only the 12 most energetic of the satellite flux spectra bins are used. A flux threshold of $10^{6}\left(\mathrm{keV} . \mathrm{cm}^{2} . \mathrm{sr} . \mathrm{s}\right)^{-1}$ has also been applied to eliminate those bins which contribute mainly noise to the spectra. In addition, a three-point running median filter has been applied to reduce noise in the satellite flux data.

Panel (E) shows the comparison between the characteristic energy derived from the ground-based energy map method (red curve) and the estimated characteristic energy from the satellite electron precipitation data (blue curve). The Maxwellian characteristic energy of the satellite electron spectra data is obtained using a least-squares fitting. The characteristic energy derived from ground-based data (red curve) is interpolated from the energy map data for each point of the spacecraft footprint in the energy map image, as shown in Figs. 2 and 3. This is necessary since the DASI data limits the temporal resolution to $10 \mathrm{~s}$, whereas the satellite data is $1 \mathrm{~s}$.

The mean fractional difference between the fitted Maxwellian spectra and the real satellite data (green line) is used for characterising the Maxwellian fit to the satellite data, which is given by Eq. (13). Hence, the green line is an indication of how poor the Maxwellian fit is for each point of the satellite flux spectra. A null value means that the data is a perfect Maxwellian. The black line in Panel (E) is the threshold (0.5), below which the Maxwellian fit to the satellite data is deemed acceptable.

Figure 2 shows the data set from the DMSP F15 spacecraft conjunction for 6 January $2000\left(K_{p}=3\right)$. Precipitation effects can be seen in the equatorward part of both the optical and 
absorption images. The Maxwellian fit to the satellite flux data is acceptable for $61 \%$ of the data points. For these satellite data points (with mean fractional difference $<0.5$ ), the deviation from the ground-based energy estimate is $21 \%$, on average. At 18:20:28 UT there are highly non-Maxwellian spectra in the satellite data for about $7 \mathrm{~s}$. As a result the fit is relatively poor (green curve). From about 18:20:36 UT, although the fit is better than before, the satellite and groundbased fits do not agree very well. This difference occurs when the spacecraft pass through a region where some of the riometer data points are contaminated by a scintillation source in the neighboring pixels of the image. Therefore, the ground-based method is probably in error.

Figure 3 shows the data from the DMSP F12 satellite pass for 6 December $1999\left(K_{p}=3\right)$. Again, precipitation effects are clearly seen in both the optical and absorption images. For the first half of the satellite pass, data agreement between the characteristic energy derived from the two methods is mostly good. For $30 \%$ of the data points the Maxwellian fit to the satellite flux data is relatively good (mean fractional difference $<0.5)$. For these satellite data points with acceptable Maxwellian fit, the deviation from the ground-based energy estimate is $31 \%$, on average. Starting from 17:59:36 UT the satellite data become non-Maxwellian and consequently the fit becomes relatively poor.

Satellite observations show that auroral particles can have quasi-monoenergetic energy spectra, as illustrated in Figs. 2 and 3. Evans (1974) has suggested these peaks are produced by electron acceleration due to electric fields parallel to the magnetic field. The Maxwellian distribution seems to be well suited for modelling auroras having broad incident electron spectra, such as diffuse aurora (Strickland et al., 1989). Sharp electron energy flux enhancements in the DMSP data, known as Large-Scale Electron Acceleration events (LSEAs), result from electromagnetic or electrostatic acceleration (i.e. either wave-particle interaction or potential drop), or both (Newell, 2000). "Large scale" is a relative term because at the DMSP altitudes the spacecraft's speed is $\sim 7.5 \mathrm{~km} / \mathrm{s}$; therefore, any feature observed by the satellite can be thought of as a largescale feature. The non-Maxwellian spectra in the satellite data may be examples of such events.

A strong auroral arc structure can be seen in the optical data and equatorward of the arc there is a region of absorption. Cosmic radio waves appear to be absorbed more strongly in regions adjacent to discrete auroral arcs than through the arc regions themselves (Stoker et al., 1997). The comparison between the energies shows that the agreement is good in the stronger absorption area where the satellite data also possesses a Maxwellian distribution. When the distribution fails to be Maxwellian the agreement between the characteristic energies is poor. It is also probable that the measurement uncertainty for absorption, which is $<0.1 \mathrm{~dB}$ (Kosch et al., 2001a), causes the energy map technique to fail after 17:59:40, thereby contributing to the disagreement with the satellite fit. This leads to deviations between the ground-based (red curve) and satellite (blue curve) estimates of the characteristic energy.
Figure 4 shows the satellite conjunction for 1 December $2000\left(K_{p}=1^{-}\right)$. Panels (D and E) represent satellite electron and ion precipitation data between 0.3 and $30 \mathrm{keV}$, respectively. Although there is ionospheric absorption in the IRIS data, there is relatively weak optical aurora in the DASI image. Likewise, the satellite electron flux data are relatively weak. However, a significant energy flux of precipitating protons can be seen in the ion spectra. It is obvious that agreement between the satellite data and the ground-based energy map method is poor much of the time. For $52 \%$ of the data points the Maxwellian fit to the satellite flux data has a mean fractional difference less than 0.5. However, for these satellite flux spectra with acceptable fit, the deviation from the ground-based energy estimate is more than $100 \%$, on average. Protons with energies more than $100 \mathrm{keV}$ can reach down to the $90-\mathrm{km}$ altitude causing absorption in the IRIS data. Using the ion spectra in order to determine their effect on ground-based characteristic energy is not possible here as the available DMSP ion precipitation data does not extend out to $>30 \mathrm{keV}$. However, it seems likely that the IRIS absorption data was contaminated by ion precipitation.

Typically, electron precipitation causes auroral absorption in the ionosphere, which takes place in the range of $60-100 \mathrm{~km}$. Enhanced ionisation in the atmosphere by precipitating high energy protons also causes absorption, which typically occurs at altitudes of $50-90 \mathrm{~km}$. Therefore, riometer absorption measurements may be either from electron or proton precipitation. In the data sets, which have been studied in this paper, the possibility of ion precipitation has been investigated using DMSP, ACE and GOES satellite data. The ACE satellite, orbiting at the L1 point, provides measurements of the solar wind particles whilst the GOES satellite detects the proton fluxes in geostationary orbit. There is no significant ion precipitation in the satellite data for the data sets shown in Figs. 2 and 3. This means the riometer data is due to absorption from the precipitating electrons only in these cases. This is summarised in column 5 of Table 1 . Proton precipitation is deemed to be significant if its flux is within 1-2 orders of magnitude of the electron flux.

Table 1 gives a summary overview of the satellite passes studied. The best comparison between satellite and groundbased estimates (column 8) for the Maxwellian characteristic energy of the precipitating particles depends on several factors, primarily that steady-state conditions should exist and that ion precipitation should be negligible compared to electron precipitation. The former relates directly to the basic assumption made in Eq. (7), whilst the latter adds to the total ionisation of the ionosphere, which was not taken into account when the energy map method was calibrated using EISCAT radar data. In principle, proton precipitation could be accounted for with $\mathrm{H}$ emission optical data (Galand, 2001) but, unfortunately, such measurements are not available for the present study. For the ground-based method, the riometer data is the weakest link. Scintillations cause unrealistic absorption values. The absorption is often quite small $(<0.5 \mathrm{~dB})$, increasing the uncertainty of the measurement $(<0.1 \mathrm{~dB})$. In addition, the riometer is affected by proton 


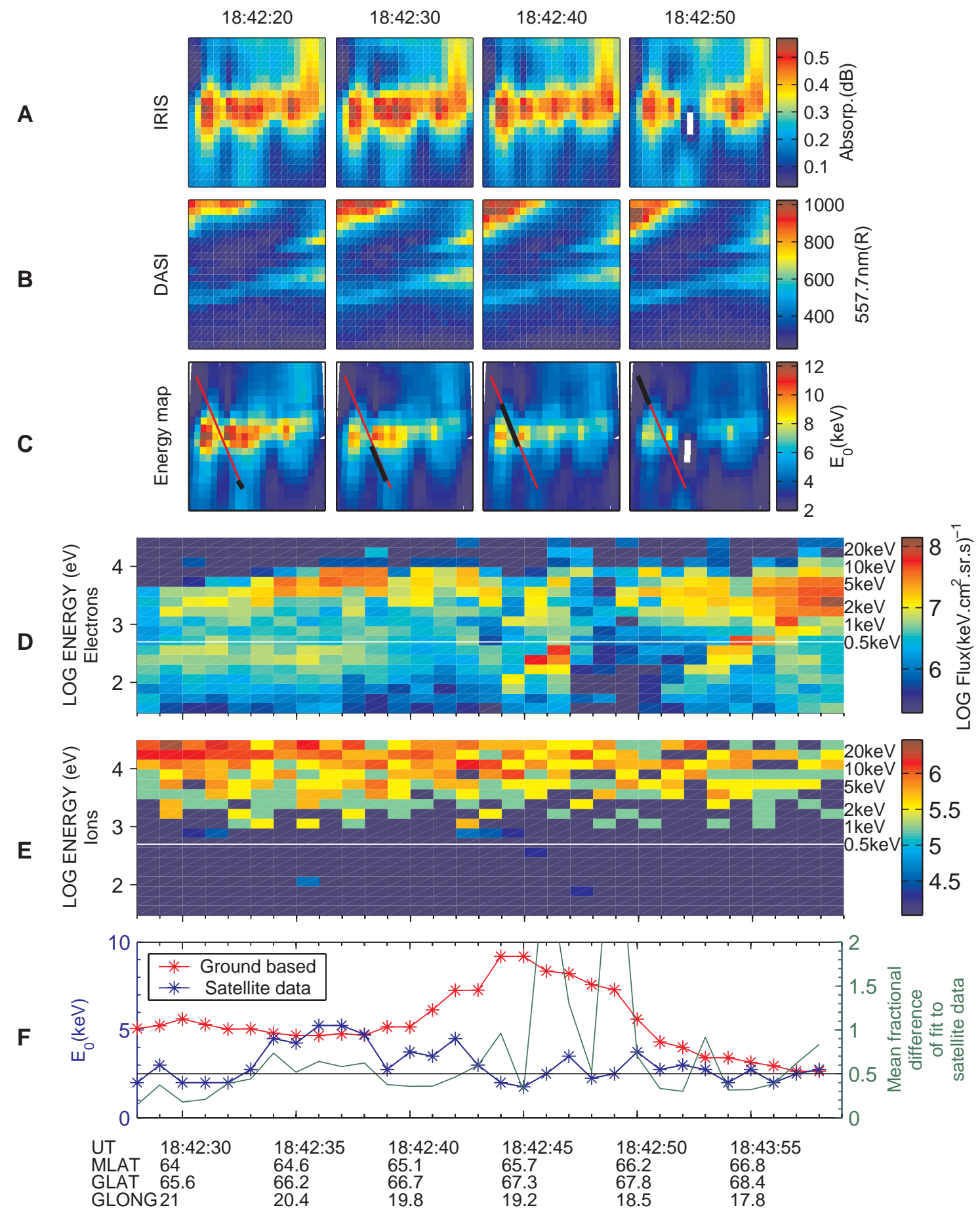

Fig. 4. Panels (A-D) are the same as Fig. 2 but for 1 December 2000, DMSP (F15) and $K_{p}=1^{-}$. Panel (E) is the satellite proton energy spectrogram. Panel (F) is the same as panel (E) in Fig. 2.

precipitation, which can only be identified optically if such an imager is available.

Five of the satellite passes (18 February 1995, 7 January 1997, 6 November 1997, 22 November 1997, 1 December 2000) occurred during non-steady-state conditions, resulting in the average discrepancy being $>100 \%$ in all cases. Two passes (19 February 1998, 28 November 2000) occurred during steady-state conditions, but the ion precipitation caused the ground-based technique to fail, also resulting in the average discrepancy being $>100 \%$ in both cases. The data related to 9 February 1997 occurred during nonsteady-state conditions and the discrepancy between satellite and ground-based methods is low (27\%), but only for a small number of data points (26\%). The data from 16 October 1996 
appears to be an anomaly since, despite ion precipitation, the number of satellite spectra under the threshold is very high $(93 \%)$ and the disagreement with ground-based energy estimates is small (35\%). However, since DMSP only has ion precipitation data for $<30 \mathrm{keV}$, it is not proven that the ions in this event did cause additional absorption.

The remaining satellite passes (6 December 1999, 6 January 2000, 23 January 2001) all occur during steady-state auroral conditions and relatively insignificant ion precipitation, and give a satisfactory agreement between satellite and ground-based energy estimates for a reasonable fraction of the data points. It is also worth noting for these data sets $K_{p}$ was moderate $\left(3-3^{+}\right)$.

\section{Conclusions}

We have reconsidered the ground-based energy map method of estimating the Maxwellian characteristic energy of the precipitating electrons and compared this to DMSP satellite observations. The results show that for moderate geomagnetic conditions with $K_{p}$ values around 3, without any significant ion precipitation present, steady-state conditions and for Maxwellian satellite spectra, there is $\sim 71 \%$ agreement between the characteristic energies derived from satellite data and the ground-based energy map method. Where the geomagnetic conditions are very quiet or disturbed there is no satisfactory agreement between the satellite electron precipitation data and the ground-based instruments' characteristic energy (Table 1). In the case of $K_{p} \gg 3$ the steady-state assumption generally fails, whereas for $K_{p}<3$ IRIS measurement uncertainties dominate. Statistical analysis shows that the average absorption decreases with decreasing $K_{p}$ (Kavanagh et al., 2004), therefore with low $K_{p}$ the possibility of small absorption resulting in greater measurement uncertainties is higher. The ground-based method works best for absorption $\gg 0.1 \mathrm{~dB}$.

One of the assumptions made in estimating the characteristic energy of precipitating particles is that of a Maxwellian flux spectrum, which is not always true. For field-aligned potential drops and particle acceleration mechanisms in the lower magnetosphere, the precipitating electron spectra can be highly non-Maxwellian (Evans, 1974). The advantage of using multi-spectral optical measurements is in estimating a characteristic energy from ground-based data, which is more general. The present study was undertaken using the atomic oxygen green line emission only, as no other optical data were available. However, future multi-wavelength optical measurements are planned. Having data from several optical wavelengths means that there is no need to assume any specific spectral distribution function.

The DASI camera is capable of recording the precipitating particles with energies $>1 \mathrm{keV}$ which is dominated by the oxygen green line at $557.7 \mathrm{~nm}$. Hence, the optical images do not include the energies $<1 \mathrm{keV}$ corresponding to F-region deposition altitudes. This energy range excites the second brightest auroral emission at $630 \mathrm{~nm}$. The long radiative lifetime of this oxygen emission results in strong quenching by collisions below $250 \mathrm{~km}$ altitude, and only electrons interacting above $\sim 250 \mathrm{~km}$ produce this emission line (Rees and Luckey, 1974). However, the 110-s radiative lifetime limits its use in studying dynamic aurora. The $\mathrm{N}_{2}^{+}$prompt emission at $427.8 \mathrm{~nm}$ is another candidate which has been used in previous auroral studies (Rees and Luckey, 1974; Strickland et al., 1989; Semeter et al., 2001). This emission is a proxy for energy flux (see Eq. 4) and can be used for studying high-energy $(>10 \mathrm{keV})$ electron precipitation in auroral forms (Semeter et al., 2001).

Applying these improvements to the energy map method provides the possibility of expanding the application regime of the energy map method of Kosch et al. (2001a) by reducing the number of constraining assumptions. In addition, imaging the proton auroral emission will remove the proton vs. electron precipitation ambiguity in the absorption data.

Acknowledgements. We thank D. Hardy, F. Rich, and P. Newell as well as JHU/APL for use of the DMSP data. IRIS is operated by the Department of Communications Systems at Lancaster University (UK) and is funded by the Particle Physics and Astronomy Research Council (PPARC) in collaboration with Sodankylä Geophysical Observatory (Finland). DASI was operated by Max-PlanckInstitut für Aeronomie (Germany) in collaboration with the Auroral Observatory in Troms $\emptyset$ (Norway). EISCAT is an international scientific association supported by the research councils of Finland, France, Germany, Japan, Norway, Sweden and the United Kingdom.

Topical Editor M. Lester thanks P. H. Stoker and another referee for their help in evaluating this paper.

\section{References}

Ansari, Z. A.: The aurorally associated absorption of cosmic noise at college, Alaska, J. Geophys. Res., 69, 4493-4513, 1964.

Berkey, F. T.: Coordinated measurements of auroral absorption and luminosity using the narrow beam technique, J. Geophys. Res., 73, 319-337, 1968.

Brekke, A., Doupnik, J., and Banks, P.: Incoherent scatter measurements of E-region conductivities and currents in the auroral zone, J. Geophys. Res., 79, 3773-3790, 1974.

Brekke, A., Hall, C., and Hansen, T. L.: Auroral ionospheric conductances during disturbed conditions, Ann. Geophys., 7, 269280, 1989.

Browne, S., Hargreaves, J. K., and Honary, B.: An imaging riometer for ionospheric studies, Electron. Commun., 7(5), 209-217, 1995.

Campbell, W. H. and Leinbach, H.: Ionospheric absorption at times of auroral and magnetic pulsations, J. Geophys. Res., 66, 25-34, 1961.

Collis, P. N., Hargreaves, J. K., and Korth, A.: Auroral radio absorption as an indicator of magnetospheric electrons and of conditions in the disturbed auroral D-region, J. Atmos. Terr. Phys., 46, 21-38, 1984.

del Pozo, C. F., Hargreaves, J. K., and Aylward, A. D.: Ion composition and effective ion recombination rate in the night-time auroral lower ionosphere, J. Atmos. Sol. Terr. Phys., 59, 1919$1943,1997$. 
del Pozo, C. F., Kosch, M. J., and Honary, F.: Estimation of the characteristic energy of electron precipitation, Ann. Geophys., 20, 1349-1359, 2002,

\section{SRef-ID: 1432-0576/ag/2002-20-1349.}

Evans, D. S.: Precipitating electron fluxes formed by a magnetic field-aligned potential difference, J. Geophys. Res., 79, 28532858,1974

Frank, L. A. and Ackerson, K. L.: Observations of charged particle precipitation in the auroral zone, J. Geophys. Res., 76, 36123643, 1971.

Fuller-Rowell, T. J. and Evans, D. S.: Height-integrated Pedersen and Hall conductivity patterns inferred from the TIROS-NOAA satellite data, J. Geophys. Res., 92, 7606-7618, 1987.

Galand, M.: Introduction to special section: Proton precipitation into the atmosphere, J. Geophys. Res., 106, 1-6, 2001.

Gustafsson, G.: Spatial and temporal relations between auroral emission and cosmic noise absorption, Planet. Space Sci., 17, 1961-1975, 1969.

Hardy, D. A., Schmitt, L. K., Gussenhoven, M. S., Marshall, F. J., Yeh, H. C., Schumaker, T. L., Hube, A., and Pantazis, J.: Precipitating electron and ion detectors (SSJ/4) for the block 5D/flights 6-10 DMSP satellites: Calibration and data presentation, Tech. Rep. AFGL-TR-84-0317, Air Force Geophysics Laboratory, Hanscom Airforce Base, MA, 1984

Hardy, D. A., Gussenhoven, M. S., and Holeman, E.: A statistical model of auroral electron precipitation, J. Geophys. Res., 90, 4229-4248, 1985.

Hargreaves, J. K.: Auroral absorption of HF radio waves in the ionosphere: A review of results from the first decade of riometry, Proceedings of the IEEE, 57, 1348-1373, 1969.

Hargreaves, J. K.: The Solar-Terrestrial Environment, Cambridge University Press, 1995.

Heppner, J. P., Byrne, E. C., and Belon, A. E.: The association of absorption and Es ionisation with aurora at high latitudes, J. Geophys. Res., 57, 121-134, 1952.

Holt, O. and Omholt, A.: Auroral luminosity and absorption of cosmic radio noise, J. Atmos. Terr. Phys., 24, 467-474, 1962.

Johansen, O. E.: Variations in energy spectrum of auroral electrons detected by simultaneous observation with photometer and riometer, Planet. Space Sci., 13, 225-235, 1965.

Kavanagh, A. J., Kosch, M. J., Honary, F., Senior, A., Marple, S. R., Woodfield, E. E., and McCrea, I. W.: The statistical dependence of auroral absorption on geomagnetic and solar wind parameters, Ann. Geophys., 22, 877-887, 2004,

SRef-ID: 1432-0576/ag/2004-22-877.

Kosch, M. J.: The Skibotn CCD All-Sky Imager (SCASI) and real time networking onto the World Wide Web, Tech. Rep. MPAET-010-99-12, Max-Planck-Institut für Aeronomie, Lindau, Germany, 1999.

Kosch, M. J., Hagfors, T., and Nielsen, E.: A new digital all-sky imager for optical auroral studies in conjunction with the Scandinavian twin auroral radar experiment, Rev. Sci. Instrum., 69, 578-584, 1998a.

Kosch, M. J., Hagfors, T., and Schlegel, K.: Extrapolating EISCAT Pedersen conductances to other parts of the sky using groundbased TV auroral images, Ann. Geophys., 16, 583-588, 1998 ,

SRef-ID: 1432-0576/ag/1998-16-583.

Kosch, M. J., Honary, F., del Pozo, C. F., Marple, S. R., and Hagfors, T.: High-resolution maps of the characteristic energy of precipitating auroral particles, J. Geophys. Res., 106, 28925 $28937,2001 \mathrm{a}$.
Kosch, M. J., Scourfield, M. W. J., and Amm, O.: The importance of conductivity gradients in ground-based field-aligned current studies, Adv. Space Res., 27, 1277-1282, 2001b.

Newell, P. T.: Reconsidering the inverted-V particle signature: The relative frequency of large-scale electron acceleration events, J. Geophys. Res., 105, 15 779-15 794, 2000.

Ogilvie, K. W.: Auroral electron energy spectra, J. Geophys. Res. 73, 2325-2332, 1968.

Rees, M. H.: Auroral ionisation and excitation by incident energetic electrons, Planet. Space Sci., 11, 1209-1218, 1963.

Rees, M. H.: Physics and Chemistry of the Upper Atmosphere, Cambridge University Press, 1989.

Rees, M. H. and Luckey, D.: Auroral electron energy derived from ratio of spectroscopic emissions, 1. Model computations, J. Geophys. Res., 79, 5181-5186, 1974.

Robinson, R. M. and Vondrak, R.: Validation of techniques for space based remote sensing of auroral precipitation and its ionospheric effects, Space Sci. Rev., 69, 331-407, 1994.

Robinson, R. M., Vondrak, R. R., Miller, K., Babbs, T., and Hardy, D.: On calculating ionospheric conductances from the flux and energy of precipitating electrons, J. Geophys. Res., 92, 25652569, 1987.

Rostoker, G., Kamide, Y., and Winningham, J. D.: Energetic particle precipitation into high-latitude ionosphere and the auroral electrojets, 3. Characteristics of electron precipitation into the morning sector auroral oval, J. Geophys. Res., 90, 7495-7504, 1985.

Semeter, J., Lummerzheim, D., and Haerendel, G.: Simultaneous multispectral imaging of the discrete aurora, J. Atmos. Sol. Terr. Phys., 63, 1981-1992, 2001.

Seviour, R., Kosch, M., and Honary, F.: Identification of clouds and aurorae in optical data images, New Journal of Physics, 5, 6.1-6.7, 2003

Stauning, P.: Investigations of ionospheric radio wave absorption process using imaging riometer techniques, J. Atmos. Terr. Phys., 58, 753-764, 1996

Steele, D. P. and McEwen, D. J.: Electron auroral excitation efficiencies and intensity ratios, J. Geophys. Res., 95, 1032110336, 1990.

Stoker, P. H., Mathews, M. J., and Scourfield, M. W. J.: Cosmic radio noise absorption related to structures in auroral luminosity, J. Geophys. Res., 102, 7439-7447, 1997.

Störmer, C.: The Polar Aurora, Oxford University Press, 1955.

Strickland, D. J., Meier, R. R., Hecht, J. H., and Christensen, A. B.: Deducing composition and incident electron spectra from ground-based auroral optical measurements: Theory and model results, J. Geophys. Res., 94, 13 527-13 539, 1989.

Vallance-Jones, A. V.: Auroral spectroscopy and thermosphere, in: Auroral Physics, edited by: Meng, C. I., Rycroft, M. J., and Frank, L. A., Cambridge University Press, 15, 1991

Vickery, J. F., Vondrak, R. R., and Matthews, S. J.: The diurnal latitudinal variations of auroral zone ionospheric conductivity, J. Geophys. Res., 86, 65-75, 1981.

Vondrak, R. R. and Sears, R. D.: Comparison of incoherent scatter radar and photometric measurements of the energy distribution of auroral electrons, J. Geophys. Res., 83, 1665-1667, 1978. 